\title{
Scrub Typhus in a Tertiary Care Hospital in Visakhapatnam
}

\author{
Vandana Shankar1 ${ }^{1}$ Mandarapu Roopalatha², Sudha Lakshmi ${ }^{3}$ \\ ${ }^{1}$ Consultant and HOD, Department of Microbiology, Indus Hospitals, Visakhapatnam, Andhra Pradesh, India. \\ ${ }^{2}$ Consultant and HOD, Department of Biochemistry, Indus Hospitals, Visakhapatnam, Andhra Pradesh, India. \\ ${ }^{3}$ Senior Technician, Department of Microbiology, Indus Hospitals, Visakhapatnam, Andhra Pradesh, India.
}

\section{ABSTRACT}

\section{BACKGROUND}

Scrub typhus is a serious public health problem in the Asia-Pacific area including, India. It is a zoonotic disease and usually presents with acute febrile illness along with multi-organ involvement caused by Orientia tsutsugamushi. It is transmitted to humans by the bite of the larvae of trombiculid mites (Chiggers) which feed on forest and rural rodents. The mites are both vector and natural reservoir. Human infection follows a chigger bite. Incubation period is 6- 21 days. Onset of scrub typhus is characterised by fever, headache, myalgia, cough and gastrointestinal symptoms. A primary lesion which later crusts to form a flat black eschar may be present which is pathognomonic feature of scrub typhus. If the condition is not treated, serious complications may occur involving various organ systems. Owing to potential for severe complications, diagnosis and decision to initiate treatment should be based on clinical suspicion and confirmed by serological tests. The recommended treatment for scrub typhus is doxycycline. Our aim was to study the magnitude of scrub typhus antibodies among febrile patients for a period of one year in a tertiary care hospital and to assess clinical features and outcomes of serologically positive patients.

\section{METHODS}

Blood was collected aseptically from 131 patients with request for investigation of scrub typhus from various departments. Screening for scrub typhus was done by rapid Immunochromatographic test to identify antibodies.

\section{RESULTS}

During the 1-year study period, a total of 131 serum samples presenting with fever were tested for scrub typhus. Of these, 46 samples were found seropositive. Among these patients, Eschar was found only in $4.3 \%$ of cases. In one patient tuberculosis and in 3 patients dengue was found as co-infection. Seasonally the highest number of cases were observed in the cooler months of the year. Male to female ratio was not significant. The percentage of patients positive for scrub typhus was highest $(17.6 \%)$ in the age group $16-25$ years followed by $(15.4 \%)$ in $26-35$ year. None among the age group of 0-15 year was positive.

\section{CONCLUSIONS}

Prompt diagnosis, timely antimicrobial therapy and intensive supportive care are important to prevent life-threatening complications. Scrub typhus detected by immunochromatography can be utilized as part of initial investigation to guide the clinicians. In the present study we found that our results highlight that scrub typhus infection is an important cause of fever and active surveillance is necessary to access exact magnitude and distribution of the disease.

\section{KEY WORDS}

Scrub Typhus, Orientia tsutsugamushi, Trombiculid Mites, Chiggers, Eschar, Zoonosis
Corresponding Author: Dr. Vandana Shankar, Flat No. 810, Lansum Green Apartments, Madhavadhara, Visakhapatnam-530007, Andhra Pradesh, India.

E-mail: vandanashankar@yahoo.co.in

DOI: $10.14260 / j e m d s / 2019 / 658$

Financial or Other Competing Interests: None.

How to Cite This Article:

Shankar V, Roopalatha M, Lakshmi S. Scrub typhus in a tertiary care hospital in Visakhapatnam. J. Evolution Med. Dent. Sci. 2019;8(40):3028-3031, DOI:

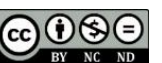




\section{BACKGROUND}

This infection is caused by Orientia tsutsugamushi, which is transmitted to humans by the bite of infected chiggers (larvae) of trombiculid mites. There are still many unknowns regarding the mechanisms of pathogenesis and the cell biology of the interaction of this bacterium with its host cell, due to the extra research obstacles of studying an obligately intracellular bacterium.(1) The disease is widespread extending from Japan to Australia and from India to pacific. The scrub Typhus continues to be a public health problem in Asia.(2) Scrub typhus in India it has been documented from Jammu and Kashmir, Himachal Pradesh, Uttaranchal, Rajasthan, Assam, West Bengal, Maharashtra, Kerala, and Tamil Nadu. After an incubation period of 6 to 21 days (Mean 10 to 12 days), symptoms of scrub typhus start suddenly and include fever, chills, headache, and generalized lymphadenopathy. At onset of fever, an eschar often develops at the site of the chigger bite. The typical lesion of scrub typhus, common in whites but rare in Asians, which begins as a red, indurated lesion about $1 \mathrm{~cm}$ in diameter; it eventually vesiculates, ruptures, and becomes covered with a black scab. Regional lymph nodes enlarge.(3) Clinical manifestations range from asymptomatic to severe disease. The mortality rate varies and can be as high 50\%.(4) Misdiagnosis and under diagnosis is known to occur due to lack of availability of diagnostic tests and the specific nature of symptoms, especially when the characteristic eschar is not present.(5),(6),(7),(8) Complications such as pneumonitis, meningoencephalitis, jaundice, renal failure, and myocarditis may also develop. The severity of infection ranges from mild features to multi organ failure and death, which occurs in around $4 \%$ of the patients presenting to a hospital. The lung is one of the main target organs for Orientia, leading to pulmonary complications of variable severity. Interstitial pneumonia may occur in severe cases.(9),(10) In our study period of one year (July 2107 to July 2018), 131 patients presenting with fever were tested for scrub typhus. Out of these 46 samples were positive. In one patient tuberculosis and in 3 patients, dengue was found as co infection. The present study was conducted to determine the magnitude of scrub typhus antibodies among febrile patients and to determine the seasonality, clinical features and outcome in patients with scrub typhus admitted in a tertiary care hospital in Visakhapatnam (AP). An eschar at the site of chigger feeding is a classic clinical feature of scrub typhus. It begins as a papule at the site of chigger feeding and then ulcerates and forms a black crust like a skin burn from a cigarette. When present, it occurs prior to the onset of fever and other symptoms.(11),(12) The case fatality can be up to 30 $70 \%$ if no appropriate treatment is received while the median case fatality rate for untreated patients is $6 \%$ and for treated

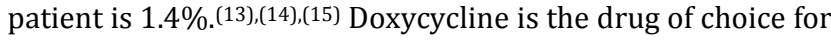
this illness. Azithromycin has been advocated as an alternative agent.

\section{METHODS}

The study was conducted in the department of microbiology in Indus Hospital Visakhapatnam for a period of one year from July 2017 to July 2018 for test of Scrub typhus specific antibodies. Requisition for testing from treating consultant is taken as IEC clearance and consent from patient. Blood was collected aseptically from 131 in-patients with request for investigation of scrub typhus from various departments. These were the patients having febrile illness of more than 5 days duration. Screening for scrub typhus was done by rapid Immunochromatographic test to identify antibodies. This rapid test (SD Bioline Tsutsugamushi) detects total IgM, IgG or IgA antibodies in human serum against 0 . tsutsugamushi. The test was performed as per recommendation of manufacturer. Briefly, $10 \mu \mathrm{l}$ serum was applied to sample well and three drops of assay diluent were added. Results were read after 10-15 min. Test had two-coloured lines, a control line " $\mathrm{C}$ " and test line "T". Colour band observed only at " $\mathrm{C}$ " was recorded as negative while if observed at both " $\mathrm{C}$ " and " $\mathrm{T}$ " recorded as positive. Absence of " $\mathrm{C}$ " line indicated invalid result.

\section{RESULTS}

During the 1-year study period, a total of 131 serum samples were received in the Department of Microbiology with the request to test for scrub typhus specific antibodies. Of these, 46 samples $(35.11 \%)$ tested positive for the presence of antibodies against 0 . tsutsugamushi. Among the samples tested negative for scrub typhus, two were found to be malaria positive, in seven cases blood cultures were positive for bacteria and remaining were suspected as having viral fevers. The number of adult male and female positive patients were $49 \%$ and $51 \%$ respectively. No paediatric samples were tested. The month-wise distribution of serologically positive cases is depicted in table 2. A retrospective analysis of the clinical presentation of the serology positive patients found that fever and headache with a mean duration of approximately 5 days was the predominant symptom. The presence of an eschar, though characteristic of scrub typhus was detectable in only $4.3 \%$ of patients. Other clinical findings included hepatosplenomegaly and lymph node enlargement. $1 / 3$ rd of patients had complications such as acute respiratory distress syndrome, hypotension, acute kidney failure and neurological manifestations such as headache, loss of sensorium and seizures with meningeal signs in varying combinations. Fever was the common symptom. In one patient tuberculosis, and in 3 patients, dengue was associate infection. Out of 46 positive cases, leucocytosis was present in 6 patients, raised blood urea in 13 patients, raised creatinine levels in 8 patients, platelet counts were low in 18 patients and liver enzymes were raised in $90 \%$. During the course of the study, one patient expired due to cardiac arrest and two patients left against medical advice. All the other serologically positive patients were treated with doxycycline and showed remarkable improvement.

\begin{tabular}{|c|c|c|}
\hline Complications & No. of Patients & Percentage \\
\hline Leucocytosis & 6 & $13.0 \%$ \\
\hline Elevated Blood urea & 13 & $28.2 \%$ \\
\hline Low Platelet counts & 18 & $39.1 \%$ \\
\hline Elevated Liver enzymes & 41 & $89.1 \%$ \\
\hline Elevated Creatinine levels & 8 & $17.4 \%$ \\
\hline \multicolumn{2}{|c|}{ Table 1. Percentage of Associated Complications } \\
\hline
\end{tabular}




\begin{tabular}{|c|c|c|c|}
\hline Month & No. of Samples Tested & No. of Positives & Monthly \% \\
\hline July, 2017 & 06 & Nil & Nil \\
\hline August, 2017 & 21 & 07 & $33.3 \%$ \\
\hline September, 2017 & 23 & 06 & $26 \%$ \\
\hline October, 2017 & 16 & 07 & $43.7 \%$ \\
\hline November, 2017 & 07 & 04 & $57.1 \%$ \\
\hline December, 2017 & 09 & 02 & $22.2 \%$ \\
\hline January, 2018 & 06 & 01 & $16.6 \%$ \\
\hline February, 2018 & 06 & 01 & $16.6 \%$ \\
\hline March, 2018 & 02 & Nil & Nil \\
\hline April, 2018 & 05 & 01 & Nil \\
\hline May, 2018 & 10 & 04 & $10 \%$ \\
\hline June, 2018 & 09 & 01 & $94.4 \%$ \\
\hline July, 2018 & 11 & $\mathbf{4 6}$ \\
\hline Total & $\mathbf{1 3 1}$ & \multicolumn{3}{|c|}{} \\
\hline \multicolumn{4}{|c|}{ Table 2. Percentage of Seasonal Occurrence } \\
\hline
\end{tabular}

\section{DISCUSSION}

Scrub typhus is an emerging public health problem and an important cause of PUO. Failure of timely diagnosis and treatment can lead to significant morbidity and mortality. This infection is caused by Orientia tsutsugamushi, which is transmitted to humans by the bite of infected chiggers (Larvae) of trombiculid mites. There are still many unknowns regarding the mechanisms of pathogenesis and the cell biology of the interaction of this bacterium with its host cell, due to the research obstacles in studying an obligate intracellular bacterium. (16)

Classically known as a post monsoon disease, this seasonal occurrence of scrub typhus was a prolonged one, extending well into the month of December.(17) During our study also period of highest incidence was found to be in post monsoon season (August to November). Eschar at the site of attachment of the larval mite/chigger is considered highly suggestive of scrub typhus, but occurs in a variable proportion of patients in different studies. (18) It begins as a papule at the site of chigger feeding and then ulcerates and forms a black crust like a skin burn from a cigarette. When present, it occurs prior to the onset of fever and other symptoms. In our study typical eschar was present only in 2 cases i.e $4.3 \%$ of cases, similar rates $(4 \%-12 \%)$ also have been reported by some other Indian studies.(19),(20),(21)

The clinical manifestations of this disease vary from minimal to severe fatal illness with multi-organ dysfunction. Lung is one of the main target organs for Orientia, leading to pulmonary complications of variable severity. Interstitial pneumonia may occur in severe cases. This has been reported in another study from the Indian subcontinent.(22)In our study $1 / 3^{\text {rd }}$ of patients had complications such as acute respiratory distress syndrome, hypotension, acute kidney failure and neurological manifestations. Kumar et al found nearly half of their patients (48.5\%) had three or more organ systems involved, while 20 patients $(30 \%)$ had evidence of dysfunction of five organs systems during the course of their hospital stay. The case fatality rate for scrub typhus has been 7\%-30\%.(23)

Mortality for scrub typhus ranges for less than $1 \%$ to $50 \%$ depending on antibiotic treatment and status of individual infected. (24) In our study there was no causality found due to scrub typhus. Thrombocytopenia is an important feature of Scrub Typhus, dengue, malaria and leptospirosis. This was presented by about half of our patients. Leucocytosis was seen in 36 per cent patients. Significantly elevated levels of one or more liver enzymes which is commonly observed in
Scrub Typhus, were also found in our study. The haematological and biochemical parameters were comparable with other reports from India and abroad. (25),(26),(27),(28)

The Weil-Felix test (WFT) is most common and commercially available test for the diagnosis of scrub typhus in developing countries like India. The sensitivity and specificity of the WFT is low and is usually positive during the $2^{\text {nd }}$ week of illness. The gold standard confirmatory tests are the indirect immune peroxidase test and the immunefluorescent assay (IFA). These tests are costly and not easily available in developing countries like India. Therefore, the diagnosis of scrub typhus is mainly by clinical suspicion and by characteristic clinical finding of, eschar. Therefore, thorough search for eschar over all the areas of body is very important during the clinical examination of all acute febrile illnesses. The current treatment for scrub typhus is Doxycycline and Chloramphenicol. Azithromycin has also been found effective.(29)

\section{CONCLUSIONS}

A high index of clinical suspicion, prompt diagnosis and early institution of appropriate antimicrobials can decrease the morbidity and mortality. Scrub typhus is easily treatable disease. Focus should be on case identification, public education, rodent control and habitat modification to control the impact of Scrub typhus. Vaccines are under trial. Detection of scrub typhus by immunochromatography can be utilized as part of initial investigation to guide the clinicians. In the present study we found that scrub typhus infection is an important cause of fever and active surveillance is necessary to access exact magnitude and distribution of the disease.

\section{REFERENCES}

[1] Giengkam S, Blakes A, Utsahajit $P$, et al. Improved quantification, propagation, purification and storage of the obligate intracellular human pathogen Orientia tsutsugamushi. PLoS Neglected Tropical Diseases 2015;9(8):e0004009.

[2] Jin HS, Chu C, Han DY. Spatial distribution analysis of scrub typhus in Korea. Osong Public Health Res Perspect 2013;4(1):4-15.

[3] Petri WA Jr. Tsutsugamushi Disease: Mite-Borne Typhus: Tropical Typhus, University of Virginia School of Medicine --MSD and the MSD Manuals, July 2018.

[4] Hu J, Tan Z, Ren D, et al. Clinical characteristics and risk factors of an outbreak with scrub typhus in previously unrecognized areas, Jiangsu Province, China 2013. PLoS One 2015;10(5):e0125999.

[5] Chikeka I, Dumler JS. Neglected bacterial zoonoses. Clin Microbiol Infect 2015;21(5):404-15.

[6] Sundriyal D, Kumar N, Chandrasekharan A, et al. Eschar: an important clue to diagnosis. BMJ Case Rep 2013;2013:010105. 
[7] Kundavaram AP, Jonathan AJ, Nathaniel SD, et al. Eschar in scrub typhus: a valuable clue to the diagnosis. J Postgrad Med 2013;59(3):177-8.

[8] Park JH, Kim SJ, Youn SK, et al. Epidemiology of scrub typhus and the eschars patterns in South Korea from 2008 to 2012. Jpn J Infect Dis 2014;67(6):458-63.

[9] Jeong YJ, Kim S, Wook YD, et al. Scrub typhus: clinical, pathologic and imaging findings. Radiographics 2007;27(1):161-72.

[10] Song SW, Kim KT, Ku YM, et al. Clinical role of interstitial pneumonia in patients with scrub typhus: a possible marker of disease severity. J Korean Med Sci 2004;19(5):668-73.

[11] Mahajan SK. Scrub typhus. J Assoc Physicians India 2005;53:954-8.

[12] Gupta V, Gautam V. Scrub typhus-a short review. J Commun Dis 2004;36(4):284-9.

[13] Bonell A, Lubell Y, Newton PN, et al. Estimating the burden of scrub typhus: a systematic review. PLoS Negl Trop Dis 2017;11(9): e0005838.

[14] Varghese GM, Abraham OC, Mathai D, et al. Scrub typhus among hospitalised patients with febrile illness in South India: magnitude and clinical predictors. Journal of Infection 2006;52(1):56-60.

[15] Taylor AJ, Paris DH, Newton PN. A systematic review of mortality from untreated scrub typhus (Orientia tsutsugamushi). PLoS Negl Trop Dis 2015;9(8):e0003971.

[16] Sharma PK, Ramakrishnan R, Hutin YJ, et al. Scrub typhus in Darjeeling, India: opportunities for simple, practical prevention measures. Trans R Soc Trop Med Hyg 2009;103(11):1153-8.

[17] Sharma N, Biswal M, Kumar A, et al. Scrub typhus in tertiary care hospital in North India. Am J Trop Med Hyg 2016;95(2):447-51.

[18] Oaks SC Jr, Ridgway RL, Shirai A, et al. Scrub Typhus: Bulletin No. 21. Institute Medical Research Malaysia,
United States Army Medical Research Malaysia, United States Army Medical Research Unit, 1983;21:1-98.

[19] Rajoor UG, Gundikeri SK, Sindhur JC, et al. Scrub typhus in adults in a teaching hospital in north Karnataka, 2011-2012. Ann Trop Med Public Health 2013;6(6):6147.

[20] Mahajan SK, Kashyap R, Kanga A, et al. Relevance of Weil-Felix test in diagnosis of scrub typhus in India. J Assoc Physicians India 2006;54:619-21.

[21] Mathai E, Rolain JM, Verghese GM, et al. Outbreak of scrub typhus in southern India during the cooler months. Ann N Y Acad Sci 2003;990:359-64.

[22] Chrispal A, Boorugu H, Gopinath KG, et al. Scrub typhus: an unrecognized threat in South India-clinical profile and predictors of mortality. Trop Doct 2010;40(3):12933.

[23] Iqbal N, Viswanathan S, Remalayam B, et al. Pancreatitis and MODS due to scrub typhus and dengue co-infection. Tropical Medicine and Health 2012;40(1):19-21.

[24] Kelly DJ, Richards AL, Temenak J, et al. The past and present threat of rickettsial disease to military medicine and international public health. Clinical Infection Disease 2002;34(Suppl 4):S145-69.

[25] Isaac R, Varghese GM, Mathai E, et al. Scrub typhus: prevalence and diagnostic issues in rural southern India. Clin Infect Dis 2004;39(9):1395-6.

[26] Vivekanandan M, Mani A, Priya YS, et al. Outbreak of scrub typhus in Pondicherry. J Assoc Physicians India 2010;58:24-8

[27] Digra SK, Saini GS, Singh V, et al. Scrub typhus in children: Jammu experience. JK Sci 2010;12:95-7.

[28] Chanta C, Chanta S. Clinical study of 20 children with scrub typhus at Chiang Rai regional hospital. J Med Assoc Thai 2005;88(12):1867-72.

[29] Panpanich R, Garner P. Antibiotics for treating scrub typhus. Cochrane Database Syst Rev 2002;(1) :CD002150. 